\title{
Is White rice the culprit for the expanding waist line in South Indians?
}

\author{
Dr Lalitha $\mathrm{R}^{1}$ MD, PGD (Cardiff), Dr Surekha Shetty B ${ }^{2}$ MD, PGD (Boston) \\ ${ }^{I}$ Dr Lalitha R Consultant Diabetologist, Karnataka Institute of Endocrinology and Research, Bannerghatta \\ Road, $9^{\text {th }}$ block Jayanagar, Bangalore560069, India, \\ ${ }^{2}$ Dr Surekha B Shetty, Consultant Diabetologist,. Karnataka Institute of Endocrinology and Research, \\ Bannerghatta Road, ${ }^{\text {th }}$ block Jayanagar, Bangalore560069.
}

\begin{abstract}
Increasing prevalence of overweight and obesity in India has a direct association with the rising prevalence of obesity-related co-morbidities. Dietary factors are correlated with overweight and obesity; therefore, dietary intervention could be a target for obesity prevention.

Objective: To assess the nutritional status of the diabetic population and to evaluate if white rice is contributing to the high prevalence of abdominal obesity, general obesity in the study group. Whether Waist Circumference (WC) and Waist Hip Ratio(WHR) are better indices for measuring obesity than Body mass Index(BMI) Methods: An observational study was done in a total of 23417 diabetics (9183 women, 14234 men) between the age group of 25-98 years. Body weight, height, waist and hip circumference were measured and recorded using standard measuring instruments. Classification of obesity was done using World Health Organisation (WHO) and International Diabetes Federation (IDF) criteria for BMI in Asians, while WC and WHR according to IDF criteria. Nutritional status of 3750 patients was assessed based on 24 hour dietary recall, accounting for the amount of carbohydrates, proteins and fat intake. Also the number of servings of vegetables and fruits were recorded.

Results: Prevalence of overweight and obese according to BMI (WHO) is $85 \%$ in women and $74 \%$ in men. WHR identified $89 \%$ of men with abdominal obesity compared to $76 \%$ of women. The waist circumference among men and women according to the IDF criteria $65 \%$ men $(n=9235)$ and $89 \%$ women $(n=8175)$ have a WC above the normal. This proves that WHR and WC are better indices than BMI in identifying people with abdominal obesity and high risk for cardiovascular morbidity. The age incidence of diabetes was similar in both men and women. Major calories are derived from carbohydrates up to the extent of $80 \%$ of which $50 \%$ is derived from white rice thus contributing to the higher prevalence of both abdominal and general obesity. In people with abnormal WC they were consuming 300-400 calories derived from white rice. The high caloric white rice adds to the burden of abdominal obesity and general obesity. $52 \%$ were consuming higher than $2000 \mathrm{k}$ calories of their daily intake. The protein consumption is only $8.5 \%$ of the total calories and this clearly indicates that there is protein deficiency in the population. $63 \%$ consumed less than 2 servings of vegetables against the recommended 3-4 servings. And 66\% did not consume any fruits at all. The mean protein intake in the population is $8.53 \%$ against the recommended $20-30 \%$.

Conclusion: High intake of carbohydrate rich diet, with major contribution from the white rice, low fibre, and low protein, inadequate intake of fruits and vegetables, lack of adequate physical activity is contributing to very high prevalence of abdominal obesity and general obesity. WC and WHR are better indices in defining obesity in the population. We need to change the practice of consumption of white rice to a healthy option of unpolished rice or brown rice, with whole grains and adequate fruits and vegetables.
\end{abstract}

Keywords: Abdominal Obesity, Body Mass Index, General Obesity, Waist circumference, White Rice

\section{Introduction}

Obesity is a worrisome crisis in the developed and developing economies. ${ }^{1}$ Obesity is one of the most universal, yet amongst the most neglected, community health problems in both developed and developing countries. ${ }^{2}$ Evidence is emerging that obesity-associated illnesses, across regions and ethnicities. ${ }^{3}$ Obesity is emerging as an important health issue particularly in urban areas, paradoxically co-existing with under nutrition. Nearly 30-65\% of adult urban Indians are either overweight or obese or have abdominal obesity. The increasing prevalence of overweight and obesity in India has a direct association with the rising prevalence of obesityrelated co-morbidities; hypertension, the metabolic syndrome, dyslipidaemia, type 2 diabetes mellitus (T2DM) and cardiovascular disease (CVD). Asian Indians appear to have higher morbidity at lower cut-off for waist circumference than do White Caucasians. BMI, abdominal fat distribution, and weight gain are important risk factors for the development of type 2 diabetes. It is estimated that $90 \%$ of patients with type 2 diabetes are obese. $^{4}$ 
Due to the increased threat of morbidity and mortality, obesity is now being known as a disease in its own right. Visceral obesity is linked with elevated triglycerides; low high-density lipoprotein (HDL) cholesterol; and increased small, dense low-density lipoprotein (LDL) particles. In adults, overweight and obesity are defined based on body mass index (BMI), which is determined as weight $(\mathrm{kg})$ divided by height ${ }^{2}$ $(\mathrm{m})^{5}$

Obese individuals particularly those with abdominal fat distribution, are at increased risk for coronary artery disease. ${ }^{6}$ Risk for these disorders appears to start from a body mass index (BMI) of about $21 \mathrm{~kg} / \mathrm{m} 2$. Obesity is generally classified as generalized obesity (GO) and abdominal obesity (AO). Obese individuals have higher rates of mortality and morbidity compared to non obese individuals. ${ }^{7,8}$

Under-nutrition due to poverty which dominated in the past is being rapidly replaced by obesity associated with affluence. Industrialization and urbanization also add to increased prevalence of obesity. Studies from different regions of India have provided evidence of the rising prevalence of obesity. ${ }^{9-12}$

The rates of coronary disease has hastened dramatically amongst South Asians, driven to an important extent by the atherogenic dyslipidaemia and type 2 diabetes that have become so common amongst them. These precursors of vascular disease emerge at lower absolute amounts of adipose tissue in South Asians than in whites. ${ }^{13}$ Increase in the prevalence of diabetes, coronary heart disease (CHD), and stroke in India points to the occurrence of an epidemiologic transition whereby, because of urbanization, there is a higher prevalence of traditional cardiovascular disease risk factors such as obesity, physical inactivity, tobacco use, and high intake of saturated fat. ${ }^{14}$

Dietary factors are correlated with overweight and obesity; therefore, dietary intervention could be a target for obesity prevention. High consumption of fibre found in fruit, vegetable and whole grain has important role in dietary interventions. High consumption of whole grain in the diet, lead to lower energy intake, decrease hunger and raise satiety. Most of the grains are consumed after removing the outer layers and just starch rich endosperm remains. Whole grains consumption is associated with a reduction in developing many metabolic diseases such as diabetes, cardiovascular disease, stroke and cancers, refined grains because of their high quantity of carbohydrates at least partly are responsible for current obesity problem. Carbohydrate on the whole important source of energy in the diet associated with postprandial blood glucose. Glycaemic index (GI) proves the ability of carbohydrate foods in raising the postprandial blood glucose. Consumption of low GI foods contributes to reduce body fat and control obesity. ${ }^{15}$

Rice is a central component in South Indian diet, and the form of rice used in a given household is often an indicator of socioeconomic status. White rice is one of the most essential sources of energy and carbohydrate. Generally, rice has been processed and refined. During refining procedure, bran and germ are eliminated and just starchy endosperm remains in white rice. Having white rice causes an increase in postprandial blood glucose as compared with brown rice. ${ }^{15}$

\section{Aims and objectives:}

This study proposed to assess the nutritional status of the diabetic population, prevalence of abdominal and general obesity. To evaluate if white rice is culprit for to the prevalence of abdominal obesity in addition to general obesity in the diabetic population.

\section{Methods}

It is an observational study held at Karnataka Institute of Diabetology, Bengaluru, during the period of 2011-2015. A total of 23417 diabetics (9183 women, 14234 men) between the age group of 25-98 years were studied. Only confirmed type 2 diabetes subjects were included. Body weight was measured using a digital scale with daily calibration with Tanita scale. Height was measured using Seca calipers; waist and hip circumference were measured using a flexible measuring tape. Height was measured in metres and weight in kilograms. Waist measurement was done in erect posture at horizontal position just above the iliac crest at the end of normal expiration in a fasting state. Hip measurement was done using a measuring tape at the widest part of the hips.

Nutritional assessment was done in 3750 patients with a 24 hours dietary recall. The total intake of carbohydrates from all sources divided as white rice and other carbohydrates, proteins, fat, vegetables and fruits were recorded in total. Classification of obesity was done using WHO and IDF criteria for BMI in Asians, while Waist Circumference and Waist Hip Ratio (WHR) according to IDF criteria.

Appropriate statistical analysis was used for the analysis of results. Pearson R, Spearman R, Correlation graph with $\mathrm{R}$ values was used to derive at the statistical significance.

\section{Anthropometric Analysis:}

According to the BMI, 2, 13, 31\& $54 \%$ women and 3, 23, 48, \& $26 \%$ men were lean, normal, overweight and obese respectively. Refer figure 1 and 2 below. 


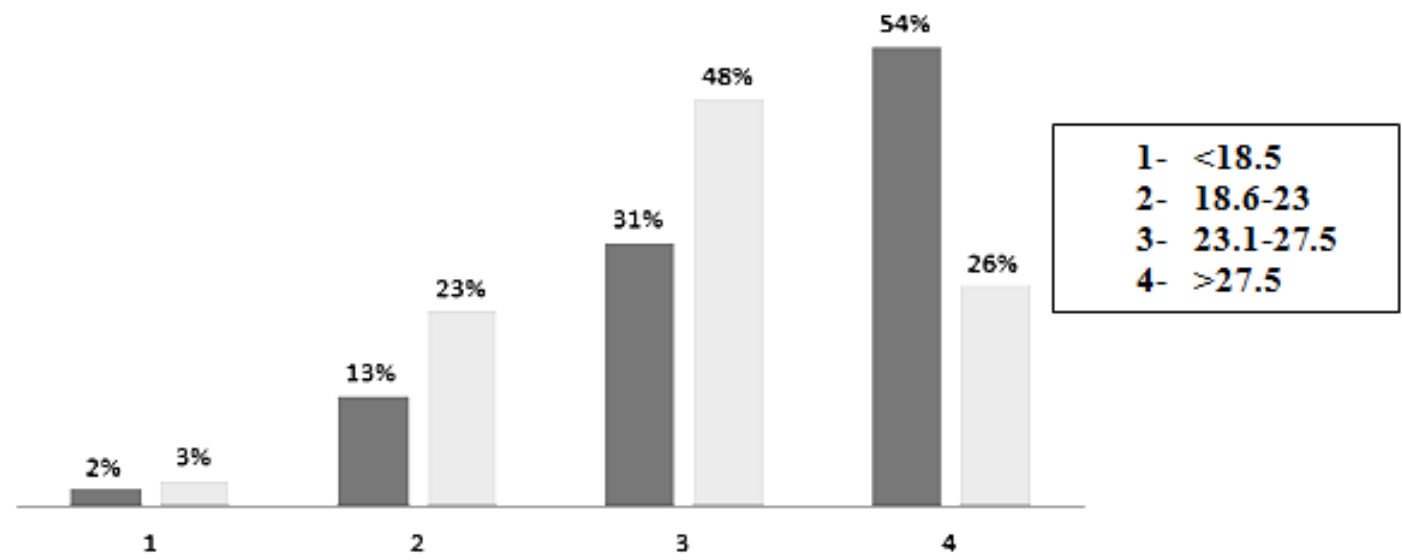

Figure 1

Comparing the waist circumference among men and women (fig 2) according to the IDF criteria $65 \%$ men $(\mathrm{n}=9235)$ and $89 \%$ women $(\mathrm{n}=8175)$ have a WC above the normal.
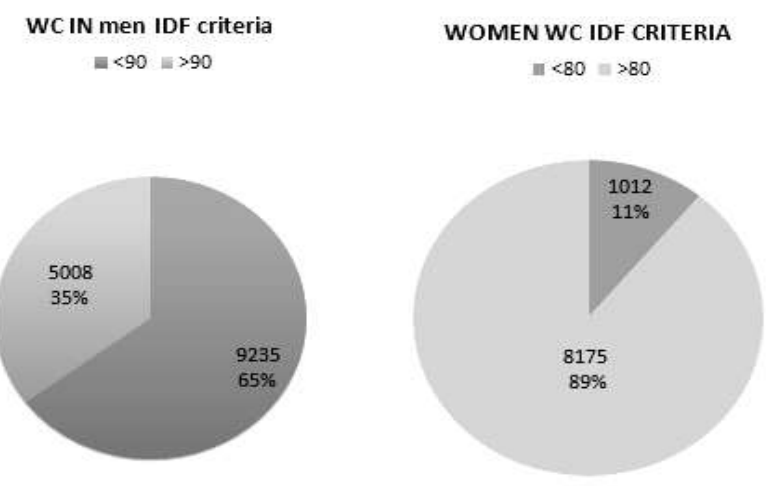

Figure 2

Figure 3 shows the difference in the waist hip ratio of men and women. Men had higher abnormal WHR (IDF criteria) compared to women. This shows that men are more obese in contrast to women.

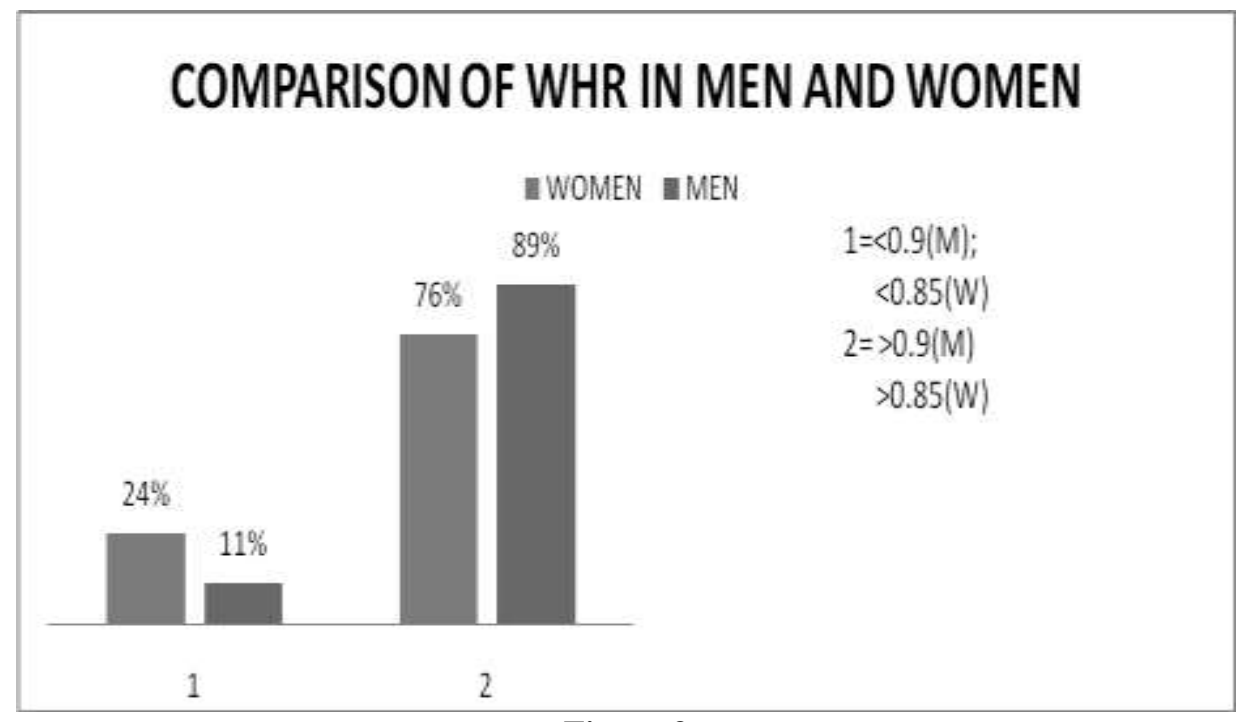

Figure 3 
In figure 3, among women, $6974(76 \%)$ had Waist Hip ratio (WHR) > 0.85 and among men, 12609 (89\%) had WHR $>0.9$. The WC was $>80 \mathrm{~cm}$ in $89 \%$ women and $>94 \mathrm{~cm}$ in $48 \%$ men.

Prevalence rates of obesity calculated according to the BMI (WHO Criteria) in men and women was $26 \%$ and $54 \%$ respectively. The age wise prevalence is similar in both groups with $58 \%$ of men and $64 \%$ of women in the age group of 46-65 years. Incidence of diabetes between men and women shows no difference among different age groups as seen in figure 4 .

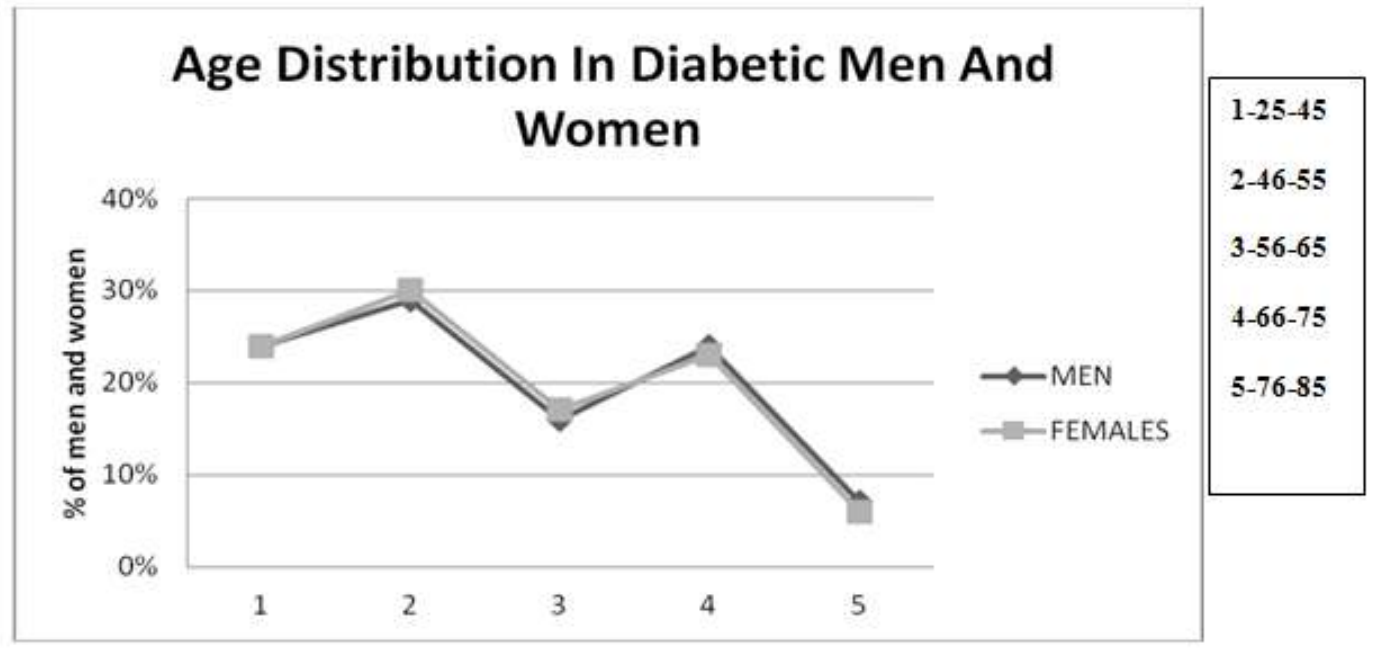

Figure 4

Nutritional Analysis: The standard recommendation from the National Nutrition society of India ,300 grams of cooked rice for daily consumption in three divided meals for man weighting $60 \mathrm{~kg}$ is considered. In the study subjects, (figure 5) more than $74 \%$ consume more than 300 grams of cooked rice and $28 \%$ of these are consuming more than 600 grams of rice.

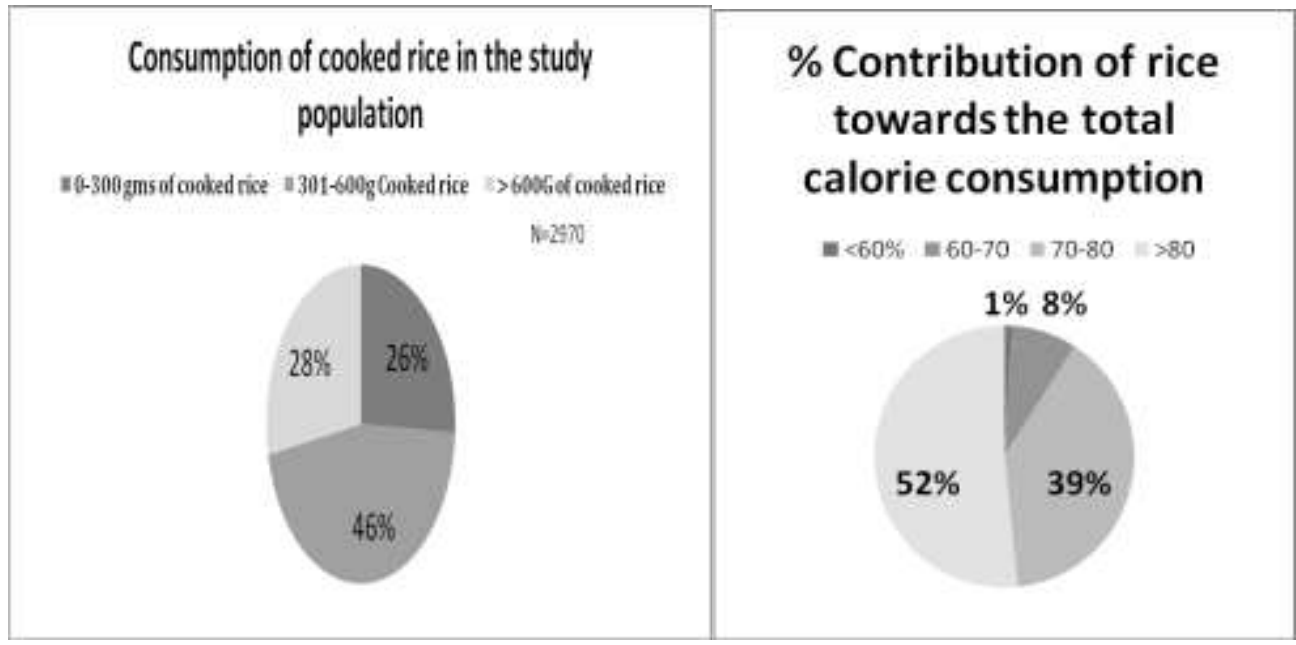

Figure 5

In the figure 5 the contribution of rice to the total calories are shown. We can see that more than $91 \%$ of the study group derived $>70$ percent of the calories from rice.

TABLE 1

\begin{tabular}{|l|l|l|l|l|}
\hline & $\begin{array}{l}\text { \% calories from } \\
\text { rice }\end{array}$ & $\begin{array}{l}\text { \%calories from } \\
\text { other cereals }\end{array}$ & $\begin{array}{l}\text { \%calories from } \\
\text { proteins }\end{array}$ & \% calories from fat \\
\hline Number of values & 2970 & 2970 & 2970 & 2970 \\
\hline Minimum & 0.0 & 0.0 & 3.000 & 4.000 \\
\hline 25\% Percentile & 27.00 & 31.50 & 6.000 & 9.000 \\
\hline Median & 36.00 & 42.00 & 8.000 & 12.00 \\
\hline 75\% Percentile & 48.00 & 54.00 & 10.00 & 15.00 \\
\hline Maximum & 89.00 & 83.00 & 20.00 & 32.00 \\
\hline
\end{tabular}


Is White rice the culprit for the expanding waist line in South Indians?

\begin{tabular}{|l|l|l|l|l|}
\hline Mean & 36.91 & 42.20 & 8.535 & 12.38 \\
\hline Std. Deviation & 18.59 & 18.76 & 3.011 & 4.384 \\
\hline Std. Error of Mean & 1.079 & 1.089 & 0.1747 & 0.2544 \\
\hline Lower 95\% CI of mean & 34.79 & 40.05 & 8.191 & 11.88 \\
\hline Upper 95\% CI of mean & $\mathbf{3 9 . 0 4}$ & $\mathbf{4 4 . 3 4}$ & $\mathbf{8 . 8 7 9}$ & $\mathbf{1 2 . 8 8}$ \\
\hline
\end{tabular}

From the above analysis (Table-1) we can see the distribution of the calories from rice, other cereals, proteins and fat towards the total calories. The mean for rice is 36.91 and mean for other cereals is 42.20 and 50 $\%$ of the study group lie in the $75 \%$ percentile, clearly indicates that the diabetic populations in spite of nutritional counseling are still consuming larger portion of rice.

TABLE 2

\begin{tabular}{|l|l|l|l|}
\hline \multicolumn{4}{|c|}{ Total daily calories consumed by a person } \\
\hline$<1600 \mathrm{k}$ cal & $1600-1999$ & $2000-2999$ & $3000-5000$ \\
\hline $20 \%$ & $28 \%$ & $48 \%$ & $4 \%$ \\
\hline
\end{tabular}

TABLE-3

\begin{tabular}{|l|l|}
\hline Calories from other cereals vs. calories from rice \\
\hline Spearman r & \\
\hline r & -0.3323 \\
\hline $95 \%$ confidence interval & -0.4326 to -0.2238 \\
\hline P value & \\
\hline P (two-tailed) & $<0.0001$ \\
\hline P value summary & $* * * *$ \\
\hline Exact or approximate P value? & Approximate \\
\hline Significant? (alpha $=0.05)$ & Yes \\
\hline
\end{tabular}

In Figure 7 we can see that more than $50 \%$ of the study group is consuming more than $2000 \mathrm{~K}$ Calories per day. A sedentary to moderately active persons requires $1600-2000$ calories per day. Clearly the excess calories are derived mainly from carbohydrates and particularly from white rice. A comparative analysis for rice versus other cereals shows a significant $\mathrm{p}$ value of 0.0001 , with $50 \%$ calories derived from white rice

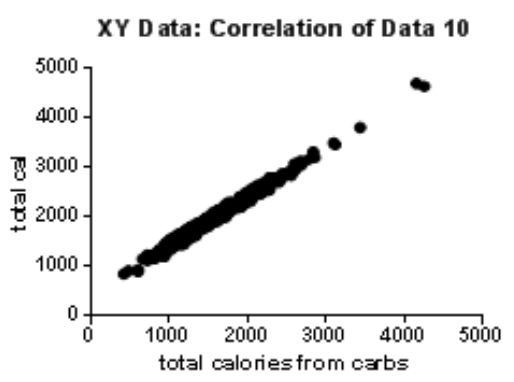

$$
\begin{aligned}
& \text { The Correlation graph shows a linear increase in } \\
& \text { the consumption of calories. More than } 80 \\
& \text { percent of total calories are derived from } \\
& \text { carbohydrates of which the rice contributes } \\
& \text { more than fifty percent. }
\end{aligned}
$$

\section{Figure 7}

In Table 4 the direct correlation from carbohydrates versus total calories derived with an $\mathrm{R}$ squared of 0.9 and significant $\mathrm{p}$ value of $<0.0001$. Also the calories derived from rice is directly related to the total calories of $\mathrm{R}$ squared $0.23 .23 \%$ variation is directly related to the calories from rice.

TABLE 4

\begin{tabular}{|l|l|l|l|}
\hline \multicolumn{2}{|l|}{ Calories from carbohydrates vs, total Calories } & \multicolumn{2}{l|}{ Calories from rice vs total calories } \\
\hline Pearson r & & Pearson $\mathrm{r}$ & \\
\hline r & 0.9919 & r & 0.4818 \\
\hline $\mathbf{9 5 \%}$ confidence interval & 0.9901 to 0.9934 & $95 \%$ confidence interval & 0.3999 to 0.5560 \\
\hline R squared & 0.9839 & R squared & 0.2321 \\
\hline P value & & P value & \\
\hline P (two-tailed) & $<0.0001$ & P (two-tailed) & $<0.0001$ \\
\hline P value summary & $* * * *$ & P value summary & $\star \star \star \star$ \\
\hline Significant?(alpha $=\mathbf{0 . 0 5 )}$ & Yes & Significant?(alpha $=0.05)$ & Yes \\
& & & \\
\hline
\end{tabular}


In the study we compared the calorie consumption from rice in normal WC and abnormal WC. Normal WC consumes 300-400 calories less from the rice compared to those with abnormal WC. (Table 5)

\section{TABLE 5}

\begin{tabular}{|l|l|l|}
\hline \multicolumn{3}{|l|}{ Comparison of calories derived from rice in Normal vs. abnormal WC } \\
\hline & Calorie from rice in normal WC & Calorie from rice abnormal WC \\
\hline Number of values & 770 & 3210 \\
\hline Minimum & 0 & 0 \\
\hline $25 \%$ Percentile & 360 & 480 \\
\hline Median & 720 & 720 \\
\hline $75 \%$ Percentile & 860 & 1080 \\
\hline Maximum & 2160 & 2200 \\
\hline $10 \%$ Percentile & 144 & 180 \\
\hline $90 \%$ Percentile & 1084 & 1440 \\
\hline Mean & 685.3 & 784.1 \\
\hline
\end{tabular}

Out of this carbohydrate load nearly 40 - 52\% came from white rice. And rest came from other cereals like wheat, Ragi and Jowar.

In figure 8 we can see the consumption of fruits in the study population. $66 \%$ of the population did not consume any fruit. Only $9 \%$ consume the standard recommended 2 servings per day. One serving is equivalent to 100 grams of fruit or vegetable.

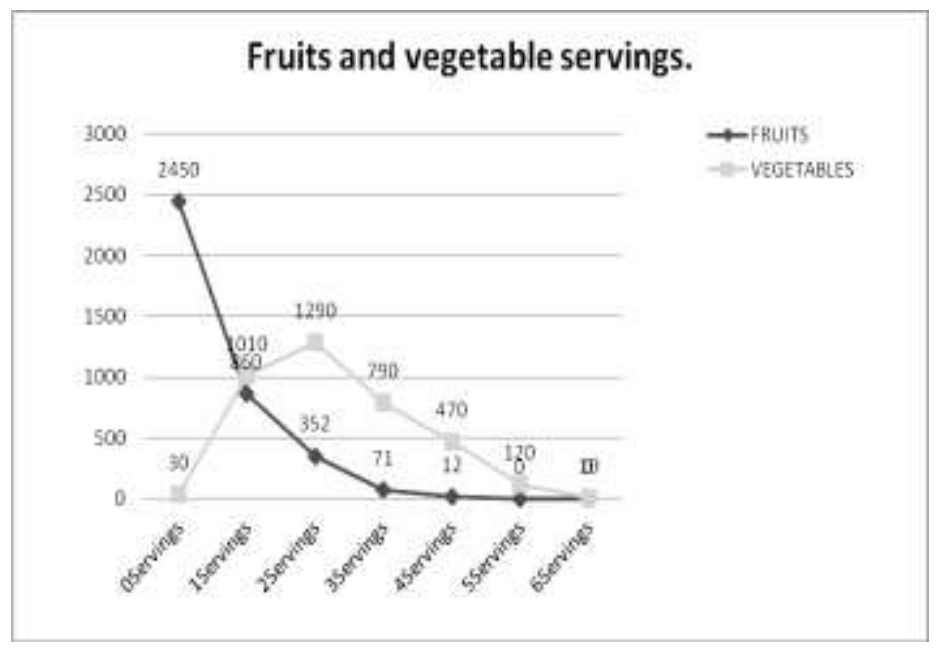

Figure 8

$62 \%$ of the populations were consuming less than 2 servings of vegetables per day.

In the study subjects only $38 \%$ were physically active (1410) and 62\% were sedentary. Among those active only 832 were moderately active.

\section{Results}

The prevalence of obesity according to BMI (WHO criteria) is $54 \%$ in women and $26 \%$ in men, which is nearly twice that for men whereas lean diabetes is $2 \%$ and $3 \%$ respectively. $76 \%$ women and $89 \%$ men had abnormal WHR and $86 \%$ women and $46 \%$ men had abnormal WC. Hence, WHR and WC identify larger number of men with abdominal obesity compared to BMI.

Twofold men were found to be overweight than obese $48 \%$ and $26 \%$ respectively and nearly twofold women were obese than overweight $54 \%$ and $31 \%$ respectively. Women had more general obesity in contrast to men.

A selected portion from this group underwent a nutritional analysis to look for the cause of abdominal obesity and general obesity. We have seen that major calories are derived from carbohydrates up to the extent of $91 \%$ of which $50 \%$ is derived from white rice. A direct correlation with a linear increment is seen with carbohydrate consumption with $\mathrm{R}$ square value of 0.9 . The protein consumption is only $8.5 \%$ of the total calories and this clearly indicates that there is protein deficiency in the population.

In the study we compared the normal WC and found that these people consumed 300 to 400 less calories derived from the rice than those with abnormal WC. Out of the carbohydrate load nearly 40-52\% was derived from white rice and rest came from other cereals like wheat, Ragi and Jowar. This high caloric white rice adds to the burden of abdominal obesity and general obesity. $52 \%$ were consuming more than 2000k 
calories of their daily intake. Added to this there is gross deficiency in the protein, vegetable and fruit intake and lack of fibre in the diet.

$63 \%$ consumed less than 2 servings of vegetables against the recommended 4 servings. And 66\% did not consume any fruits at all. The mean protein intake in the population is $8.53 \%$ as against the recommended $20 \%$. The physical activity too is poor in the study group with only $38 \%$ being active and only $50 \%$ of the active do $>150$ minutes of the recommended weekly exercise.

All these factors are contributing to the rising obesity levels and high incidence of diabetes in the south Indian population.

\section{Discussion}

Obesity and overweight can be a particularly potent risk factor for Asian Indians and, under a revised case definition for obesity of a BMI >23, an epidemic of obesity is underway in urban India and is emerging in rural India. Underlying this epidemic is a complex nutrition transition in vegetarians in which whole plant foods (fruit, vegetables, nuts, seeds, unrefined whole grains) are being replaced by refined carbohydrates. The National Family Health Survey-3 (NFHS-3) also reported that in India, obesity (BMI $\geq 25 \mathrm{~kg} / \mathrm{m}^{2}$ ) was more prevalent in the urban areas and in higher socio-economic groups compared to the rural areas, especially among women. Our study also showed the prevalence of obesity according to BMI (WHO criteria) is $54 \%$ in women and $26 \%$ in men, which is nearly twice that of men. ${ }^{16}$ Looking at the prevalence of abdominal obesity men have higher WHR compared to women. This shows that BMI is not the true indicator of obesity for people with diabetes or at risk for diabetes.

Physical activity was inversely related to abdominal obesity and variables related to health care expenditure. Similarly, in diabetic patients, physical activity is inversely associated with expenditure on medication for the treatment of diseases other than diabetes It is well documented that physical activity is related to the prevention of many diseases such as obesity, dyslipidaemia and hypertension as well early-mortality. This study showed physical activity too is poor in the study group with only $38 \%$ being active and only $50 \%$ of the active achieving more than 150 minutes of the recommended weekly exercise. ${ }^{17}$

Low-protein, high-fat group stored more than $90 \%$ of their extra calories as fat, and the lack of dietary protein caused their loss of lean body mass. The protein consumption is only $8.5 \%$ of the total calories and this clearly indicates that there is protein deficiency in the population.

South Indian population is known to consume white rice as their staple diet. Rice is consumed with three meals a day. Major portion of the meal is rice. A typical day starts with breakfast which is mainly rice in various forms and mid day meal consists of rice and dinner also ends with rice in the form of steamed rice, boiled rice or other forms of rice dishes. The quantity of intake of vegetables and fruits was recorded as servings per day. Each serving is $100 \mathrm{~g}$ of fruits and vegetables. The recommended standards as per the National Nutrition Society of India are $60-70 \%$ of carbohydrates, 20 per cent proteins and 10-15\% of fat, vegetables $4-5$ servings with fruits of 2-3servings per day.

In a study by Majid Kolahdouzan etal, ${ }^{15}$ stated white rice consumption causes an increase in postprandial blood glucose and could be a probable reason for obesity. The high caloric white rice adds to the burden of abdominal obesity and general obesity. $52 \%$ were consuming more than $2000 \mathrm{k}$ calories of their daily intake in our study.

\section{Conclusion}

In this study we have seen the high prevalence of abdominal obesity with general obesity in the diabetic people with nutritional deficits in the group. We can conclude that the high intake of carbohydrate calorie rich diet, with major contribution from white rice, low fibre, and low protein, inadequate intake of fruits and vegetables and lack of physical activity is contributing to high prevalence of abdominal obesity and general obesity. This abdominal obesity can have adverse effects on the morbidity and mortality by increase in the risk of cardiovascular mortality and micro vascular complications.

In order to change this trend measures to increase the intake of unpolished rice or brown rice, adequate proteins, fibre rich whole grains, fruit and vegetables. This can be achieved by proper nutritional counseling from point of initial contact, by the treating physicians and team of nutritionists, nurses, through media and other mass communication.

Ethical responsibilities of authors: The manuscript has not been submitted to any other journal, has not been published previously, and has not been fabricated or manipulated.

Conflict of interest: The authors disclose no potential conflict of interest.

Informed consent: Informed consent was obtained from all individual participants included in the study. Acknowledments: I thank the nutritionists, Ms Sharanya Shetty, Ms Shruthi, Ms Deepthi, Ms Shilpa and Mr Pradeep B K for their help in contributing to this paper. 


\section{References}

[1]. Stein CJ. The epidemic of obesity Clin Endocrinol Metab. 2004; 89:2522-5.

[2]. World Health Organization (WHO). Obesity: preventing and managing the global epidemic. Report of a WHO consultation. World Health Organ Tech Rep Ser 2000; 894: i-xii, 1-253.

[3]. Lau DC. The Obesity Canada Clinical Practice Guidelines Steering Committee and Expert Panel. Synopsis of the 2006 Canadian clinical practice guidelines on the management and prevention of obesity in adults and children. Can Med Assoc J. 2007;176:11036

[4]. Allison DB, Saunders SE. Obesity in North America. An overview. Med Clin North Am 2000;84:305-32,

[5]. Flegal KM, Carroll MD, Ogden CL, et al. Prevalence and trends in obesity among US adults, 1999-2008. JAMA 2010; 303: 23541.

[6]. Eckel RH, Krauss RM. American Heart Association call to action: obesity as a major risk factor for coronary heart disease. AHA Nutrition Committee. Circulation 1998;97: 2099-100

[7]. Flegal KM, Kit BK, Orpana H, Graubard BI. Association of 4. All-cause mortality with overweight and obesity using standard body mass index categories: a systematic review and meta-analysis. JAMA 2013; 309: 71-82.

[8]. World Health Organization (WHO). Global health risks: 5. mortality and burden of disease attributable to selected major risks. Geneva: Switzerland, WHO; 2009. Available from: http://www.who.int/healthinfo/global_burden_disease/Global Health Risks_ report_full.pdf, accessed on February 3, 2014.

[9]. Mohan V, Deepa R. Obesity \& abdominal obesity in Asian 6. Indians. Indian J Med Res 2006; 123: 593-6.

[10]. Bhardwaj S, Misra A, Misra R, Goel K, Bhatt SP, Rastogi 7. KV, et al. High prevalence of abdominal, intra-abdominal and subcutaneous adiposity and clustering of risk factors among urban Asian Indians in North India. PLoS One 2011; 6 : e24362.

[11]. Deepa M, Farooq S, Deepa R, Manjula D, Mohan V. Prevalence 8. and significance of generalized and central body obesity in an urban Asian Indian population in Chennai, India (CURES: 47). Eur J Clin Nutr 2009; 63: 259-67.

[12]. Misra A, Khurana L. Obesity and the metabolic syndrome 9. in developing countries. J Clin Endocrinol Metab 2008; 93 (11 Suppl 1): S9-30.

[13]. Allan D Sniderman, Why might South Asians be so susceptible to central obesity and its atherogenic consequences? The adipose tissue overflow hypothesis. International Journal of Epidemiology 2007;36:220-225.Pramil N Singh. Global epidemiology of obesity, vegetarian dietary patterns, and noncommunicable disease in Asian Indians Am J Clin Nutr. 2014 Jul; 100(1): 359S-364S.

[14]. Majid Kolahdouzan. The association between dietary intake of white rice and central obesity in obese adults. ARYA Atheroscler. 2013 Mar; 9(2): 140-144.

[15]. International Institute for Population Sciences (IIPS) and Macro International. National Family Health Survey (NFHS-3), 2005-06: India: vol. I. Mumbai: IIPS; 2007

[16]. Jamile S. Codogno. The burden of abdominal obesity with physical inactivity on health expenditure in Brazil. Motriz, Rio Claro, Jan./Mar. $2015.21(1): 68-74$. 